\title{
Philosophiques
}

\section{Christian Lazzeri, Force et justice dans la politique de Pascal, Paris, PUF (coll. " Philosophie d'aujourd'huil "), 1993, 360 p.}

\section{Christian Nadeau}

Volume 23, numéro 2, automne 1996

URI : https://id.erudit.org/iderudit/027410ar

DOI : https://doi.org/10.7202/027410ar

Aller au sommaire du numéro

Éditeur(s)

Société de philosophie du Québec

ISSN

0316-2923 (imprimé)

1492-1391 (numérique)

Découvrir la revue

Citer ce compte rendu

Nadeau, C. (1996). Compte rendu de [Christian Lazzeri, Force et justice dans la politique de Pascal, Paris, PUF (coll. " Philosophie d'aujourd'hui »), 1993, 360 p.]

Philosophiques, 23(2), 441-443. https://doi.org/10.7202/027410ar d'utilisation que vous pouvez consulter en ligne.

https://apropos.erudit.org/fr/usagers/politique-dutilisation/ 
Christian Lazzeri, Force et justice dans la politique de Pascal, Paris, PUF (coll. "Philosophie d'aujourd'hui "), 1993, 360 p.

Si plusieurs travaux ont démontré l'originalité de la position pascalienne sur la question politique, aucun ne l'a fait jusqu'à maintenant d'une manière aussi systématique que le travail de Christian Lazzeri. En comparaison, l'étude de Gérard Ferreyrolles (Pascal et la raison du politique, Paris, PUF, 1984) demeure une approche très partielle.

Afin d'y lire un modèle cohérent de pensée politique, Ferreyrolles s'intéresse davantage à l'œuvre polémique de Pascal, en particulier à la controverse des Provinciales : le chapitre II ( L'anomie jésuite ") a pour objet la dénonciation par Pascal de la casuistique et de la morale laxiste des jésuites. Pascal nie les propositions de la casuistique pour ensuite en proposer la forme inverse. Il établit ainsi les principes positifs à la base de son discours politique. Selon Ferreyrolles, l'originalité de Pascal tient au fait de tirer les principes de viabilité des institutions politiques de cela mème qui menace la stabilité du pouvoir : la violence propre à l'ordre de concupiscence est détournée par le lẻgislateur en vue de la paix. La violence est donc garante de la force de la loi et la seule crainte qu'elle inspire s'inscrit dans l'imagination des sujets et des gouvernants. permettant ainsi au pouvoir de demeurer toujours le mēme et de réaliser les fins qui lui sont propres, soit en tout premier lieu la conservation de la paix. Mais Ferreyrolles, à l'encontre de Lazzeri, semble faire peu de cas de ce qui, chez Pascal, fait de lui un penseur politique essentiellement critique. En effet, l'intérèt du travail de Lazzeri - ètude rigoureuse mais d'approche difficile tient précisément en ce qu'il démontre comment l'œuvre pascalienne n'a pas pour seul contenu l'observation et l'explication des phénomènes politiques existants ; elle est d'abord et avant toute chose l'interrogation critique sur le domaine du posssible en matière de politique. L'œuvre politique de Pascal s'inscrit alors dans une perspective plus large que celle que lui assigne Ferreyrolles.

Le travail de Lazzeri est d'un intérêt capital pour tout chercheur s'intéressant à la période classique, tant pour les domaines du droit, de la politique et de la pensée religieuse de cette époque. La première partie de l'ouvrage est consacrée à l'u anthropologie "pascalienne et à la constitution de l'État. Lazzeri s'intéresse en tout premier lieu à l'augustinisme de Pascal quant à la distinction entre les deux natures de l'homme, soit l'état d'innocence de la première nature et l'état de corruption de la seconde. L'auteur explique clairement les implications politiques de la concupiscence : Pascal dênoncerait toute transgression des frontières du pensable en matière de politique. Puisque ce domaine n'a de réalité que dans la seule sphère du temporel, contingent par définition, toute prétention à l'universalité des normes ou à la légitimation du pouvoir par une autorité divine ne ferait que nier ce qui pour Pascal est l'essence même de la politique, soit la concupiscence.

La notion pascalienne de "force "est le thème principal de toute la deuxième partie de l'ouvrage de Lazzeri. L'auteur fait alors intervenir les notions plus connues d'histoire et de providence, du rōle central de l'imagination dans l'anthropologie politique de Pascal et de la critique du statut de la connaissance. S'il faut insister sur la nécessité absolue de l'approche de ces thématiques en vue d'une compréhension parfaite de la pensée politique de Pascal, il faut plutōt renvoyer le lecteur à d'autres travaux, plus détaillés (cf. Vincent Carraud, Pascal et la philosophie, PUF, 1992 ; G. Bras et J.-P. Clèro, Pascal, Figures de l'imagination, PUF, 1994). 
Une analyse rigoureuse du thème politique chez Pascal ne peut se faire sans considérer au préalable les deux questions suivantes : 1) Comment définir la notion de politique chez Pascal en tenant compte de l'ensemble du corpus de son œuvre ? 2) Guelle place doit-on accorder à la notion d'" homogénéité des trois ordres"dans ce que Lazzeri nomme, suivant en cela de nombreux commentateurs, l'n anthropologie "politique de Pascal ? Si la question politique apparaît clairement dans les Provinciales, en particulier la $14^{\mathrm{e}}$, ainsi que dans les fameux Trois discours sur la condition des grands, l'approche en est plus difficile dans les Pensées. Mais nous ne pouvons faire l'économie de la notion des "trois ordres", dont Jean Mesnard "Le thème des trois ordres dans l'organisation des Pensées ", in Pascal Thématique des pensées, Vrin. 1988) montra naguère l'importance majeure dans l'articulation du discours apologétique. Aussi, semble-t-il impossible de comprendre la première question sans avoir d'abord obtenu une réponse satisfaisante à la deuxième.

La politique est tout entière dans l'ordre des "corps " et n'appartient ni à l'ordre de la charité ni à celui de l'esprit. Mais cela ne dégage pas la question politique des considérations théologiques : la politique, comme l'" ordre "des corps, se définit d'une manière négative comme étant ce qui n'est pas de l'ordre de la charité divine. Ferreyrolles, à l'instar de Sellier (Pascal et saint Augustin, Armand Colin, 1970), avait défini la "société politique "de Pascal comme étant la conséquence directe du péché originel : la politique est l'w ordre de la concupiscence " (Ferreyrolles, op. cit., p. 101).

Plus précise, la définition de Lazzeri insiste sur le désir de domination (libido dominand $\hat{\imath}$ qui résulte du passage difficile et incomplet de la première nature de l'homme, la "nature pure ", à la seconde, la "nature corrompue". La "première nature "désigne la condition de l'humanité avant le péché originel ; la seconde désigne l'état de concupiscence. La politique n'est donc pas seulement ce qui, dans l'homme, souligne la concupiscence ; elle est aussi le résultat du désir de l'homme de retrouver sa première grandeur - celle d'avant la chute. Ce désir participe aussi au désir de domination : à la grandeur de Dieu, l'homme déchu tente de substituer sa propre grandeur et de l'imposer à autrui. L'homme est donc animé d'un amour infini pour lui-même, amour qui ne saurait être réservé dans la " pure nature "qu'à Dieu seulement. Lazzeri souligne ainsi l'importante différence entre Pascal-penseur des * deux natures "de l'homme - et la plupart des théoriciens du droit naturel.

La notion d' ordre " a son origine dans le Potestatum Numericum Summa de 1654. Il s'agit d'une étude sur la notion d'infini. La conclusion de ce texte nous enseigne que les * ordres de grandeur sont $[\ldots]$ tout à la fois incommensurables et hiérarchisés" (p. 265). Incommensurables en ce sens que le domaine d'un ordre ne saurait s'étendre à celui d'un autre; hiérarchisés, puisque les qualités des ordres diffèrent selon la proximité du divin. La fonction des ordres est " d'établir des rapports $[. .$.$] de convenance exclusifs entre des puissances$ naturelles (raison, cœur, esprit, volonté, corps) ou surnaturelles et le domaine d'objets sur lequel elles operent au moyen de règles spécifiques [...]. " (Lazzeri, p. 265). Un ordre est donc ce qui definit les modalités d'un rapport entre une puissance et son objet en fonction des exigences qu'ont respectivement l'une envers l'autre en vue de s'accorder.

La thèse de l'incommensurabilité des ordres ne contredit en rien la possibilité d'observer dans un domaine particulier l'effet conjugué de deux ordres différents. Le domaine de la connaissance est ainsi composé de trois ordres différents : le cœur pour la certitude, la raison pour l'assurance de la validité d'une proposition et le corps pour les données de l'expérience. Mais jamais les principes d'un ordre n'interviennent sur ce qui a trait à un autre ordre ; chaque 
ordre possède donc en lui-même son autonomie. La connaissance peut ellemême ètre qualifiée d'ordre et se voit ainsi distinguée de l'ordre de la théologie par exemple, puisque les ordres la composant n'y sont pas subordonnés d'une mème manière.

En plus de leur incommensurabilité et des rapports hiérachiques existant entre eux, les ordres se soumettent tous à un "rapport de justice interne " (p. 271), ce qui veut dire qu'il ne saurait y avoir d'obstacles entre une puissance et un objet d'un mème ordre: l'ordre de l'esprit exige la reconnaissance du savant, lordre de la chantê celle du Christ, etc. Il existe donc chez Pascal des "devoirs de justice"qui ne sont rien d'autre que l'expression des rapports nècessaires entre une demande et la réalisation de cette dernière dans les limites permises par la sphère des ordres. Aussi ne peutil jamais y avoir une mais plusieurs justices. ce qui explique le rejet chez Pascal d'une norme unique et universelle. Dans une note très intéressante, Lazzeri commente brièvement la réception moderne de la notion d' ordres de justice ". en particulier dans l'œuvre de $\mathrm{M}$. Walzer (Spheres of Justice, A defense of pluralism and Equality, 1983).

Lazzeri et Ferreyrolles s'intéressent à deux domaines différents de la politique pascalienne, soit l'institution du pouvoir chez Ferreyrolles et l'، anthropologie politique " décrivant les conditions de possibilité de l'institution chez Lazzeri. Il n'en demeure pas moins que l'ouvrage de ce demier laisse voir un net progrès des études pascaliennes sur ce thème depuis dix ans et renouvelle ainsi les modes d'approche des œuvres politiques du "Grand Siècle *. Un index des noms complète l'ouvrage et confirme ainsi sa valeur scientifique.

Christian Nadeau

Département de philosophie Université du guébec à Montréal 